\title{
Stability of fractional neutral systems with multiple delays and poles asymptotic to the imaginary axis
}

\author{
André R. Fioravanti, Catherine Bonnet and Hitay Özbay
}

\begin{abstract}
This paper addresses the $H_{\infty}$-stability of linear fractional systems with multiple commensurate delays, including those with poles asymptotic to the imaginary axis. The asymptotic location of the neutral chains of poles are obtained, followed by the determination of conditions that guarantee a finite $H_{\infty}$ norm for those systems with all poles in the left half-plane of the complex plane.
\end{abstract}

Keywords: Delay effects, Neutral Systems, Fractional Systems, $H_{\infty}$ stability.

\section{INTRODUCTION}

Fractional order systems are obtaining large attention in the literature in the last years. Indeed, they appear in various engineering applications, see e.g., [1], [4], [6], [9], [10], [11], [14] and their references, where stability analysis and controller design problems are studied.

Similarly, time-delay systems have a strong practical and theoretic appeal. They appear naturally in many engineering processes, as transports, communications, biological systems, among many others.

Recently, there has been a strong development of new methods dealing with fractional order system with delays. In [8], a numerical procedure based on Cauchy's integral theorem was proposed to test the stability of such systems, and in [7], a technique based on the Lambert $\mathrm{W}$ function was used for the same purpose. Moreover, in [5], the stability regions for $P I^{\lambda} D^{\mu}$ controllers for a fractional dead-time delay system were obtained by means of the D-decomposition method.

The systems of neutral type are the most difficult to analyze, since they may have chains of poles asymptotic to the imaginary axis in the complex plane. In the frequency domain, preliminary results have been obtained in the single delay case in [3] showing that $H_{\infty}$-stability might happen or not for systems having a chain of poles clustering the axis in the left half-plane. Simple necessary and sufficient conditions have been derived there.

The aim of this paper is precisely the generalization of these results to the case of neutral systems with several commensurate delays. We are first interested in section II in the precise location of chains of poles around the asymptotic axis. The case of multiple chains around the same axis is more delicate than the case of a single chain, since a higher

A. R. Fioravanti and C. Bonnet are with INRIA Saclay - Île-de-France, Parc Orsay Université, 4 rue J. Monod, 91893, Orsay Cedex, France. [Andre.Fioravanti, Catherine.Bonnet] @inria.fr

Hitay Özbay is with Bilkent University, Department of Electrical and Electronics Engineering, Ankara 06800, Turkey. hitayabilkent.edu.tr order analysis might be necessary. In section III, easy-tocheck conditions guaranteeing $H_{\infty}$-stability are derived for systems with chains of poles around the asymptotic axis. Finally several numerical examples are given in section IV.

The notation used throughout is standard. The set of natural numbers is denoted by $\mathbb{N}$, whereas $\mathbb{N}_{N}$ denotes the set of its first $N$ elements (i.e., $\mathbb{N}_{N}=\{1, \ldots, N\}$ ). The imaginary unit is $\jmath=\sqrt{-1}$.

\section{LOCATION OF POLES OF THE SYSTEM}

We consider fractional time-delay systems with transfer functions of the form

$$
G(s)=\frac{t(s)}{p(s)+\sum_{k=1}^{N} q_{k}(s) e^{-k s h}},
$$

where $h>0$, and $t, p$ and $q_{k}$, for all $k \in \mathbb{N}_{N}$, are real polynomials in the variable $s^{\mu}$ for $0<\mu<1$. Note that we define an analytic branch of $s^{\mu}$ on the cut plane $\mathbb{C} \backslash \mathbb{R}_{-}$by setting $\left(r e^{\jmath \theta}\right)^{\mu}=r^{\mu} e^{\jmath \mu \theta}$ and choosing $\theta$ with $-\pi<\theta<\pi$.

We assume that the system is of neutral type, which means that the polynomials $p$ and $q_{k}$ further satisfy $\operatorname{deg} p \geq \operatorname{deg} q_{k}$ and such that $\operatorname{deg} p=\operatorname{deg} q_{k}$ for at least one $k \in \mathbb{N}_{N}$. In order for (1) to be a proper neutral type delay system we assume also that $\operatorname{deg} p \geq \operatorname{deg} t$. Here, the degree is interpreted as the degree in $s^{\mu}$, and therefore it is an integer.

One will notice that the essence of the analysis throughout this paper is somehow independent of the numerical value of the delay. Indeed, for the characterization of the chains of poles of large modulus, only the relations between the delays are important. Hence, both systems which have fixed known delays as well as systems with unknown delays but in a known fixed ratio can be considered in this framework. Unfortunately, if the delays vary independently, even a small variation in the absolute value can generate a great variation in the ratio, and the analysis does not apply in its present form.

The stability of a system of type (1) is linked to the location of its poles. Those poles of small modulus can be found by numerical techniques, whereas for those of large modulus, the asymptotic behavior is crucial.

We write $z=e^{-s h}$ and suppose that for each $k$

$$
\frac{q_{k}(s)}{p(s)}=\alpha_{k}+\frac{\beta_{k}}{s^{\mu}}+\mathcal{O}\left(s^{-2 \mu}\right) \quad \text { as } \quad|s| \rightarrow \infty,
$$

where, denoting $\operatorname{deg} p=d, p(s)=\sum_{m=0}^{d} \rho_{m} s^{\mu m}$ and $q_{k}(s)=$ 
$\sum_{m=0}^{d} \sigma_{k, m} s^{\mu m}$, equation (2) is satisfied with

$$
\begin{aligned}
\alpha_{k} & =\frac{\sigma_{k, d}}{\rho_{d}}, \\
\beta_{k} & =\frac{\sigma_{k, d-1}-\alpha_{k} \rho_{d-1}}{\rho_{d}} .
\end{aligned}
$$

The coefficient of the highest degree term of $p(s)+$ $\sum_{k=1}^{N} q_{k}(s) e^{-k s h}$, can then be written as a multiple of the following polynomial in $z$

$$
\tilde{c}(z)=1+\sum_{i=1}^{N} \alpha_{i} z^{i}
$$

Our initial concern is to find the position of the vertical lines for which the roots of the neutral chains are asymptotic to.

Proposition 2.1: Let $G(s)$ be a neutral system defined as in (1) and consider the greatest integer $M \leq N$ such that $\alpha_{M} \neq 0$. There exist neutral chains of poles which asymptotically approach the vertical lines

$$
\operatorname{Re}(s)=-\frac{\ln (|r|)}{h}
$$

for each root $z=r$ of the polynomial $\tilde{c}(z)$

Sketch of Proof 2.1: The poles of the neutral chains of $G(s)$ are asymptotic to the roots of (5), which leads to

$$
s_{n} h \approx \lambda_{n}=-\ln (r)+2 \jmath n \pi, \quad n \rightarrow \infty \in \mathbb{Z}
$$

The case where all the roots of (5) have multiplicity one is much easier to analyze and can be completely treated. So, for now on and throughout the rest of the paper, we will consider that Assumption 2.1 holds.

Assumption 2.1: All the roots of $\tilde{c}(z)$ are of multiplicity one.

In this particular case, there will be $M$ chains of neutral poles, and since those are asymptotic to vertical lines, it is now necessary to discover on which side the actual poles lie. As it will be seen later, this is especially important for poles such that $|r|=1$, because this will provide information on which side of the imaginary axis the poles are, and this plays a crucial role in questions about stability. This analysis is the objective of the next theorem.

Theorem 2.1: Let $G(s)$ be a neutral delay system defined by (1) and suppose that all the roots of (5) have multiplicity one. For each $r$ such that $z=r$ is a solution of (5) and for large enough $n \in \mathbb{Z}$, the solutions asymptotic to (6) are given by

$$
s_{n} h=\lambda_{n}+\delta_{n}+\mathcal{O}\left(n^{-2 \mu}\right)
$$

with $\lambda_{n}$ given by (7) and

$$
\delta_{n}=\frac{h^{\mu} \sum_{k=1}^{N} \beta_{k} r^{k}}{(2 \jmath \pi n)^{\mu} \sum_{k=1}^{N} k \alpha_{k} r^{k}}
$$

Proof: We have $p\left(s_{n}\right)+\sum_{k=1}^{N} q_{k}\left(s_{n}\right) e^{-k s_{n} h}=0$. Dividing both sides by $p\left(s_{n}\right)$ leads to

$$
1+\sum_{k=1}^{N} \frac{q_{k}\left(s_{n}\right)}{p\left(s_{n}\right)} e^{-k s_{n} h}=0 .
$$

Writing $s_{n} h=\lambda_{n}+\delta_{n}+\mathcal{O}\left(n^{-2 \mu}\right)$ when $s_{n}$ is the pole near $\lambda_{n}$, noting that $e^{-\lambda_{n}}=r$, and using (2) together with the fact that $e^{-\delta_{n} k}=1-\delta_{n} k+\mathcal{O}\left(n^{-2 \mu}\right)$ leads to

$$
1+\sum_{k=1}^{N}\left(\alpha_{k}+\frac{\beta_{k}}{s_{n}^{\mu}}\right) r^{k}\left(1-\delta_{n} k\right)+\mathcal{O}\left(n^{-2 \mu}\right)=0,
$$

and hence, assuming $n$ large enough in (7)

$1+\sum_{k=1}^{N}\left(\alpha_{k}+\beta_{k}\left(\frac{h}{2 \pi j n}\right)^{\mu}\right) r^{k}\left(1-\delta_{n} k\right)+\mathcal{O}\left(n^{-2 \mu}\right)=0$

Considering the approximation up to $\mathcal{O}\left(n^{-2 \mu}\right)$, remembering that $r$ satisfies (5), we obtain

$$
\left(\frac{h}{2 \pi j n}\right)^{\mu} \sum_{k=1}^{N} \beta_{k} r^{k}-\delta_{n} \sum_{k=1}^{N} k \alpha_{k} r^{k}+\mathcal{O}\left(n^{-2 \mu}\right)=0,
$$

which completes the proof under the assumption that $r$ is a root of multiplicity one of (5).

Some conclusions can be obtained by considering $\delta_{n}$. First of all, associated with each root $r$ of (5), let us define $K_{r}$ as

$$
K_{r}=\frac{\sum_{k=1}^{N} \beta_{k} r^{k}}{\sum_{k=1}^{N} k \alpha_{k} r^{k}}
$$

where, again, $K_{r}$ is well defined as it is assumed that $r$ has multiplicity one as a root of (5)

Our interest is mainly on which side of the vertical line the poles are, in other words, to find out the sign of $\operatorname{Re}\left(\delta_{n}\right)$ for $n$ sufficiently large. Just like the case $\mu=1$, it is sufficient to look only at $K_{r}$ to obtain this information. First, let us recall that for the non-fractional case $(\mu=1)$ [2], only the existence of poles on both sides of the line could be assured up to this point, because either $\operatorname{Re}\left(\delta_{n}\right)=0$, in which case an analysis with further terms was needed, or $\operatorname{Re}\left(\delta_{n}\right)= \pm c$, with the different signs coming from the calculation of $\delta_{n}$ for the complex conjugated root $r^{*}$ of (5).

On the other hand, for our current case, as $0<\mu<$ 1 , complex conjugated roots of (5) will not always provide complex conjugated $\delta_{n}$, that means, in general, $\delta_{n}$ associated to a particular $r$ is not equal to the complex conjugate of the one associated with $r^{*}$. That means one can prove that all poles for some systems are all in one particular halfplace delimited by the vertical line given by (6) just with the approximation up to this order. This analysis will be the subject of the next two corollaries.

Corollary 2.1: Let $0<\mu<1, \delta_{n}$ be given by (9) and its associated $K_{r}$ by (14). Then, $\operatorname{sign}\left(\operatorname{Re}\left(\delta_{n}\right)\right)<0$ for all $n \in \mathbb{R}$ if and only if

$$
\operatorname{Re}\left(K_{r}\right)<-\tan \left(\frac{\mu \pi}{2}\right)\left|\operatorname{Im}\left(K_{r}\right)\right|
$$


Proof: Besides $K_{r}$, the only term of interest is $J=$ $(\jmath n)^{-\mu}$, as $\operatorname{sign}\left(\operatorname{Re}\left(\delta_{n}\right)\right)=\operatorname{sign}\left(\operatorname{Re}\left(J K_{r}\right)\right)$. Since $n$ can be both positive or negative, this term is given by

$$
J=|n|^{-\mu}(\cos (\mu \pi / 2) \pm \jmath \sin (\mu \pi / 2)) .
$$

Multiplying $J$ by $K_{r}$ and getting its real part leads to

$\operatorname{Re}\left(J K_{r}\right)=\frac{1}{|n|^{\mu}}\left(\cos \left(\frac{\mu \pi}{2}\right) \operatorname{Re}\left(K_{r}\right) \mp \sin \left(\frac{\mu \pi}{2}\right) \operatorname{Im}\left(K_{r}\right)\right)$

from where (15) follows from the fact that $0<\mu<1$.

Some aspects can be seen from this corollary. First, the numerical value of the delay does not appear explicitly in equation (15). This means that for all $h>0$ the chains of poles present the same behavior in the sense that they do not change sides with respect to the vertical line in question as a function of an increasing delay. Second, as (15) involves only the absolute value of $\operatorname{Im}\left(K_{r}\right)$, the results obtained would be equivalent if we had dealt with the complex conjugate of $K_{r}$. But indeed, it is direct to see that complex conjugated roots of the polynomial $c(z)$ in equation (5) will define complex conjugated $K_{r}$.

Therefore, as stated before, differently from the case $\mu=$ 1 , in the present context it might be possible to state if all the poles are in the left of the vertical line (6) up to this level of approximation. Indeed, as $\alpha_{k}$ and $\beta_{k}$ given in equations (3) and (4) are independent of $\mu$, the next corollary can be stated.

Corollary 2.2: Let $0<\mu<1, \delta_{n}$ be given by (9) and its associated $K_{r}$ by (14). Then, if $\operatorname{Re}\left(K_{r}\right)<0$, all poles of the respective chain asymptotic to the vertical line (6) will be on the left of this line if

$$
\mu<\frac{2}{\pi} \arctan \left(-\frac{\operatorname{Re}\left(K_{r}\right)}{\left|\operatorname{Im}\left(K_{r}\right)\right|}\right)
$$

Proof: This follows directly from corollary 2.1 .

Although some cases might still require further analysis, as for example if all $\beta_{k}$ are equal to zero, the procedure resembles the one given in [2] and therefore will be omitted. With these results in hand, in the next section we will consider the $H_{\infty}$ stability of $G(s)$.

\section{III. $H_{\infty}$ STABILITY}

We are now interested in answering the question of stability of $G(s)$. The notion on which we will concentrate is $H_{\infty}$ stability, that is, the system has a finite $L_{2}(0, \infty)$ input/output gain.

We will refer to poles in the closed right half-plane $\overline{\mathbb{C}}_{+}$ as unstable poles, and those in the open left half-plane $\mathbb{C}_{-}$ as stable poles.

The case where equation (5) possesses only roots of modulus strictly greater than one is easy to handle as there exists $a>0$ such that the system has a finite number of poles in $\{\operatorname{Re}(s)>-a\}$. Also, the case where equation (5) possesses at least one root of modulus strictly less than one is obvious, since there will be a chain of poles asymptotic to a vertical line in the right half-plane, and consequently an infinite number of unstable poles.
Proposition 3.1: Let $G(s)$ be a transfer function given as (1) and suppose that (5) has at least one simple root of modulus one, the other roots being of modulus strictly greater than one.

1) Suppose that $\operatorname{Re}\left(\delta_{n}\right)<0$ for all $n \in \mathbb{N}$ and that $G$ has no unstable pole (which could exist only in a finite number), then $G$ is $H_{\infty}$-stable if and only if $\operatorname{deg} p \geq$ $\operatorname{deg} t+1$.

2) If $\operatorname{Re}\left(\delta_{n}\right)=0$, then the condition $\operatorname{deg} p \geq \operatorname{deg} t+1$ is necessary for $H_{\infty}$-stability.

Proof: Let $s=s_{n}+\eta \in \jmath \mathbb{R}$, we have

$$
\begin{aligned}
& \left|p(s)+\sum_{k=1}^{N} q_{k}(s) e^{-k s h}\right| \\
& \approx|\eta|\left|p^{\prime}\left(s_{n}\right)+\sum_{k=1}^{N}\left(q_{k}^{\prime}\left(s_{n}\right)-k h q\left(s_{n}\right)\right) e^{-k s_{n} h}\right| \\
& \quad \approx h|\eta|\left|p\left(s_{n}\right)\right|\left|\sum_{k=1}^{N} \alpha_{k} r^{k} k\right|
\end{aligned}
$$

as $n \rightarrow \infty, n \in \mathbb{Z}$.

Recall that $\sum_{k=1}^{N} \alpha_{k} r^{k} k$ is non zero by assumption.

If $\operatorname{Re}\left(\delta_{n}\right) \neq 0$, then $\eta$ is at least of order $n^{-\mu}$ and a necessary and sufficient condition of $H_{\infty}$-stability is that $\operatorname{deg} p \geq \operatorname{deg} t+1$. If $\operatorname{Re}\left(\delta_{n}\right)=0$ the condition is still necessary.

\section{EXAMPLES}

As a first example, let us consider the following fractional delay system

$$
G_{1}(s)=\left(s^{\mu}+e^{-s}+\left(-s^{\mu}+2\right) e^{-2 s}\right)^{-1} .
$$

It is in form of (1) with $h=1$. Evaluating (2) for this system leads to $\alpha_{1}=0, \alpha_{2}=-\beta_{1}=-1$ and $\beta_{2}=2$. The two roots of the polynomial $\tilde{c}(z)$ given in (5) are $r= \pm 1$, and so, (22) has two chains of neutral poles asymptotic to the imaginary axis. The associated values of $K_{r}$ are $K_{r}=-1.5$ and $K_{r}=-0.5$ for $r=1$ and $r=-1$ respectively.

Since $\operatorname{Re}\left(K_{r}\right)<0$ and $\operatorname{Im}\left(K_{r}\right)=0$ for both roots of $\tilde{c}(z)$, this system has both its neutral chains of poles on the left of the imaginary axis for all $0<\mu<1$. This means that for all these values of $\mu$ there are only finitely many poles on the right half-plane. Interesting is to note that, applying the results of [2] for the case $\mu=1$, we find out that for this particular value, both chains are located in the right half-plane, and therefore there exist infinitely many unstable poles.

Figures (1) and (2) show these phenomena. The first one brings the location of the chains of poles for $\mu=0.9$ and the second for $\mu=0.99$. Both graphics were obtained by the use of the QPmR algorithm [16]. One can notice that by increasing $\mu<1$, although the infinitely number of poles of the chain are still located on the left half-plane, an increasing number of unstable poles will be present. This somehow agrees with equation (18), in the sense that a lower $\mu$ is advantageous when dealing with the stability of the neutral chains. 


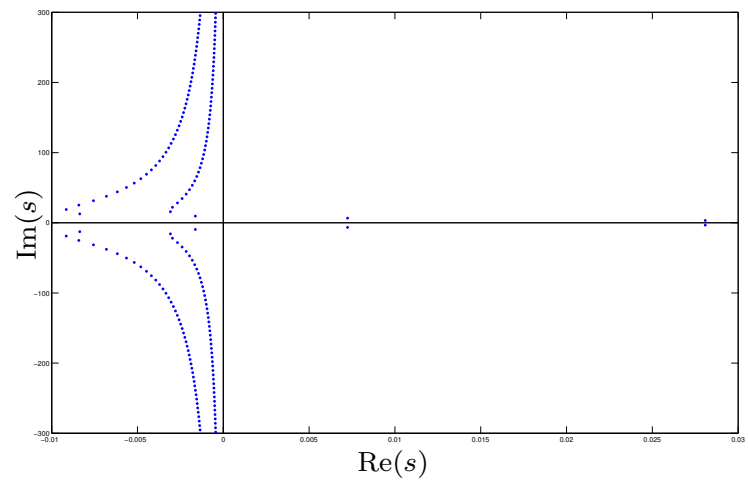

Fig. 1. Neutral Chains of Poles for $G_{1}(s)$ and $\mu=0.9$

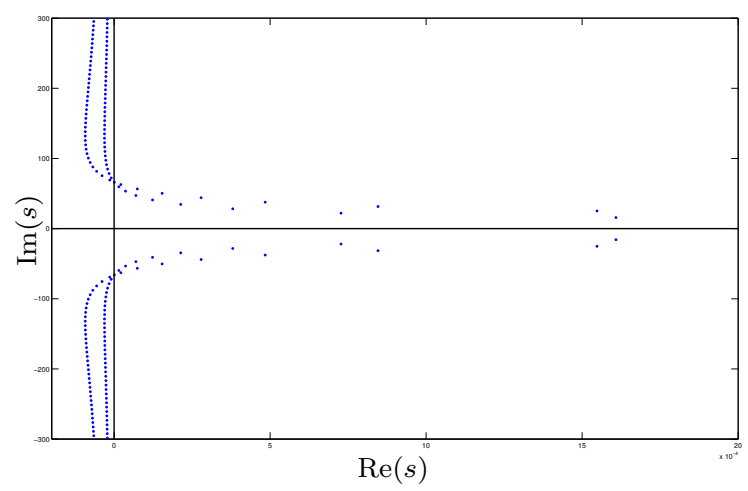

Fig. 2. Neutral Chains of Poles for $G_{1}(s)$ and $\mu=0.99$

The second example consists of the following system

$$
G_{2}=\left(s^{\mu}-s^{\mu} e^{-s}+\left(s^{\mu}-2\right) e^{-2 s}\right)^{-1} .
$$

It presents $h=1$, and its asymptotic behavior is described by $\alpha_{1}=-\alpha_{2}=-1, \beta_{1}=0$ and $\beta_{2}=-2$. The two roots of the polynomial $\tilde{c}(z)$ are $r=1 / 2 \pm \jmath \sqrt{3} /$, and therefore it also has two chains of neutral poles asymptotic to the imaginary axis. The associated values of $K_{r}$ are $K_{r}=-1 \pm \jmath 0.5774$.

Since $\operatorname{Re}\left(K_{r}\right)<0$ and $\operatorname{Im}\left(K_{r}\right) \neq 0$, we know that there exists a $\mu^{\star}$ such that for $\mu<\mu^{\star}$ the two chains of poles will be on the left of the imaginary axis. Applying equation (18), we discover that this will happen if $\mu<2 / 3$. Figure (3) brings the location of the chains of poles for $\mu=0.6$ and Figure (4) the one for $\mu=0.7$. Again, in the first image, we can see the occurrence of some poles of the stable chains already moving towards the right half-plane, creating the shape for the unstable chain that is present for $\mu=0.7$. The analysis exactly in the transition point, that is, for $\mu=2 / 3$, needs a higher order approximation in (2).

For the last example, let

$$
G_{3}(s)=\frac{t(s)}{s^{0.5}+10-\left(0.8 s^{0.5}+2\right) e^{-s}+\left(s^{0.5}-5\right) e^{-2 s}}
$$

This system has two chains of stable poles asymptotic to the imaginary axis and no unstable poles of small modulus. Figure (5) brings the Bode plot of the system for two cases of $t(s)$. In the upper part, $t(s)=s^{0.5}+1$ and in the lower part $t(s)=1$. One can see that for the first case, even with all

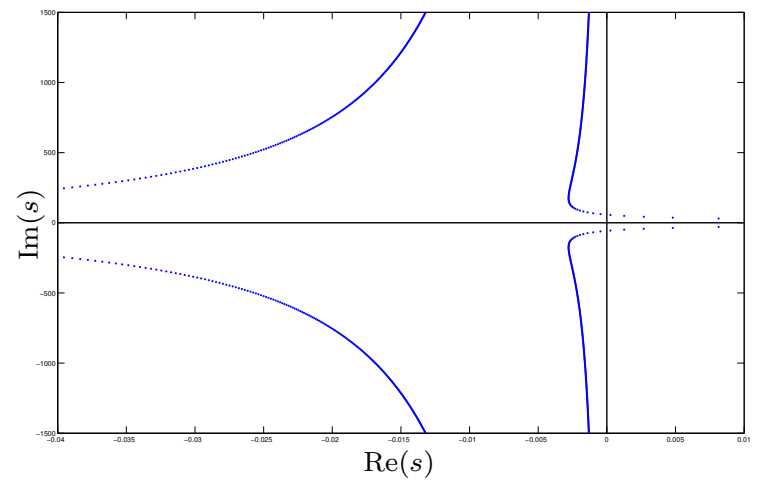

Fig. 3. Neutral Chains of Poles for $G_{2}(s)$ and $\mu=0.6$



Fig. 4. Neutral Chains of Poles for $G_{2}(s)$ and $\mu=0.7$

poles on the left half-plane, the maximum of the magnitude of the bode plot is unbounded, and therefore the system is not $H_{\infty}$ stable. On the other hand, for the second case, we have a bounded maximum value of the magnitude, and with that the $H_{\infty}$ stability of the system is achieved.

This figure illustrates the fact that the rule "No poles in the closed right half-plane" is only a necessary condition for the $H_{\infty}$ stability of a system with chains of poles asymptotic to the imaginary axis. As shown before, for this case, another necessary condition is that the relative degree between the numerator and the denominator interpreted as the degree in $s^{\mu}$, must be at least one. When these two necessary conditions are satisfied then the system is $H_{\infty}$ stable.

\section{CONCLUSion}

In this paper we have proposed some procedures to find the asymptotic behavior of the neutral chains of poles of fractional time-delay systems. This information is necessary in order to guarantee stability of systems when the chain approaches the imaginary axis. We also derived conditions for $H_{\infty}$ stability of systems with all poles in the left halfplane but chains asymptotic to the imaginary axis.

We are currently considering the problem of a design procedure of controllers for such systems.

\section{REFERENCES}

[1] H-S. Ahn, YQ. Chen, I. Podlubny, "Robust stability test of a class of linear time-invariant interval fractional-order system using Lyapunov inequality," Applied Mathematics and Computation, vol. 187 (2007), pp. 27-34. 

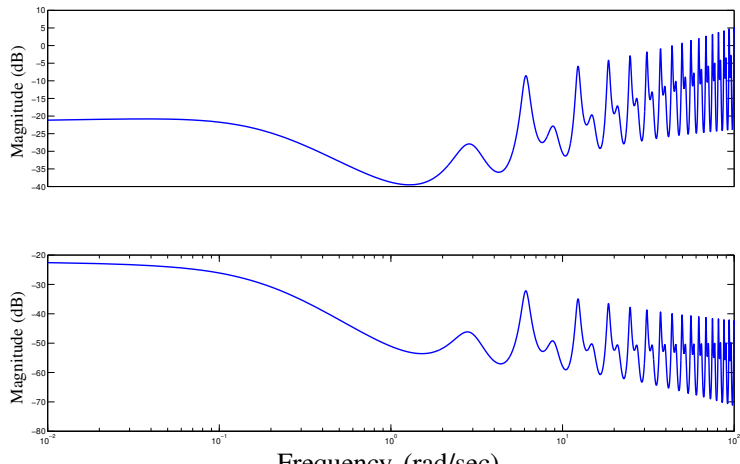

Frequency $(\mathrm{rad} / \mathrm{sec})$

Fig. 5. Bode Diagram for $G_{3}(s)$ with $t(s)=s^{0.5}+1$ and $t(s)=1$

[2] C. Bonnet, A. R. Fioravanti and J. R. Partington, "Stability of neutral systems with multiple delays and poles asymptotic to the imaginary axis", Proceedings of the Joint 48th IEEE Conference on Decision and Control and 28th Chinese Control Conference, Shanghai, P.R. China, December 16-18, 2009, pp. 269-273.

[3] C. Bonnet and J. R. Partington, "Stabilization of some fractional delay systems of neutral type," Automatica, vol. 43 (2007), pp. 2047-2053.

[4] YQ. Chen, H-S. Ahn, I. Podlubny, "Robust stability check of fractional order linear time invariant systems with interval uncertainties," Signal Processing, vol. 86 (2006), pp. 2611-2618.

[5] S. E. Hamamci, "An algorithm for stabilization of fractional-order time delay systems using fractional-order PID controllers," IEEE Transactions on Automatic Control, vol. 52(10), 2007, pp. 1964-1969.

[6] T. T. Hartley and C. F. Lorenzo, "Dynamics and Control of Initialized Fractional-Order Systems," Nonlinear Dynamics, vol. 29, (2002), pp. 201-233.

[7] C. Hwang and Y.-C. Cheng, "A note on the use of the Lambert W function in the stability analysis of time-delay systems," Automatica, vol. 41(11), 2005, pp. 1979-1985.

[8] C. Hwang and Y.-C. Cheng, "A numerical algorithm for stability testing of fractional delay systems," Automatica, vol. 42(5), 2006, pp. 825-831.

[9] R. Malti, M. Aoun, O. Cois, A. Oustaloup, F. Levron, " $H^{2}$ Norm of Fractional Differential Systems," Proceedings of ASME 2003 Design Engineering Technical Conferences and Computers and Information in Engineering Conference Chicago, IL, September 2003, DETC2003/VIB-48387.

[10] D. Matignon, "Stability Properties for Generalized Fractional Differential Systems," ESAIM Proceedings Fractional Differential Systems Models Methods and Applications vol. 5, 1998, pp. 145-158.

[11] D. Matignon, B. d'Andrea-Novel, "Observer-based controllers for fractional differential systems," Proc. of the 36th IEEE Conference on Decision and Control, San Diego, CA, December 1997, pp. 49674972.

[12] S. I. Niculescu and K. Gu, Advances in Time-Delay Systems. Springer, 2004.

[13] L. S. Pontryagin, "On the zeros of some elementary transcendental functions". Amer. Math. Soc. Transl. (1955), 2(1), 95-110

[14] A. G. Radwan, A. M. Soliman, A. S. Elwakil, A. Sedeek, "On the stability of linear systems with fractional-order elements," Chaos, Solitons \& Fractals (2007), doi:10.1016/j.chaos.2007.10.033.

[15] J. P. Richard, "Time-Delay Systems: An Overview of Some Recent Advances and Open Problems". Automatica, 39(2003), 1667-1694.

[16] T. Vyhlídal and P. Zítek, "Quasipolynomial mapping based rootfinder for analysis of time delay systems", Time Delay Systems - A Proceedings volume from the 4th IFAC workshop, Rocquencourt, France, (2003), pp. 227-232. 